Editorial

\title{
Auditory brainstem response and hearing loss in melas: post mortem analysis of temporal bone to explain abnormal ABR
}

Volume 10 Issue I - 2018

Keywords: auditory brainstem response, micrornas, sgs

Abbreviations: ABR, auditory brainstem response; SNHL, sensorineural hearing loss; PTA, pure tone audiometry; SV, stria vascularis; ROS, redox oxidative species; MELAS, myopathy, encephalopathy, lactic acidosis and stroke-like episodes

\section{Editorial}

We would like to bring to the readers' attention a specific aspect of Auditory Brainstem Response (ABR) in patients with Mitochondrial Myopathy, Encephalopathy, Lactic Acidosis and Stroke-Like Episodes (MELAS). In the literature, it is often reported that the outcome of ABR testing is normal in these patients, even when they suffer from severe to profound SensoriNeural Hearing Loss (SNHL); however, some authors observed absence of ABR waves in mild forms of SNHL. ${ }^{1-6}$ How can this disagreement in observations be explained?

We observed a MELAS patient who came to our Clinical Center for moderate SNHL. The ABR from this patient displayed waves (Figure 1) with amplitude and latency too reduced to be considered within the normal range, but not completely absent as it would be expected considering his auditory threshold in pure tone audiometry (PTA).

In an attempt to explain this observation, we reviewed the literature on the temporal bone aspect of MELAS patients. Loss of Spiral Ganglions (SGs) and atrophy of Stria Vascularis (SV) are typical findings in temporal bone of patients with MELAS even when the Organ of Corti is entirely preserved.

In SGs and SV mitochondria are abundant, ${ }^{7}$ as they are necessary to support the high metabolic functions of these structures. In MELAS, mitochondria that have been damaged (by deletion/mutation in their DNA) increase the Redox Oxidative Species (ROS) concentration ${ }^{8}$ and cell apoptosis ${ }^{9}$ which reduce the number of inner ear cells (SG, VS and Hair cells) and, cause a malfunction of the remaining cells.

ABR reflects the way the auditory signal travels from the retrocochlear portion (synapse, SGs, cochlear nerve and cochlear nuclei) (Waves I, III) to the cortex (wave V); if all the structures in the retro-cochlear hearing pathways function correctly, the ABR displays normal amplitude and latency; but damage in even one of the structures causes abnormal ABR latency and/or amplitude, which in turn alter the overall signal shape.

We think that the abnormal ABR of our patient can be explained by the altered functions of residual SGs, which cannot sufficiently amplify the signal to generate an actual ABR wave. The numerous mitochondrial mutations observed in the temporal bone of patients with MELAS ${ }^{10-11}$ support the idea that the residual SGs do not function well enough to allow a correct transmission of the impulse, which in turn leads to an abnormal ABR. Arguably, the high ABR variability in

\author{
Di Stadio Arianna,' De Lucia Antonietta, ${ }^{2}$ \\ Dipietro Laura, ${ }^{3}$ Della Volpe Antonio ${ }^{2}$ \\ 'San Camillo Hospital IRCCS, Italy \\ ${ }^{2}$ Santobono Hospital, Otolaryngology- Cochlear Implant Center, \\ Italy \\ ${ }^{3} \mathrm{Highland}$ Instruments, USA
}

Correspondence: Arianna Di Stadio, San Camillo Hospital IRCCS, Via degli Alberoni 70, Venice (IT), Italy, Email ariannadistadio@hotmail.com

Received: January 19, 2018 | Published: January 22, 2018

MELAS patients reported in the literature ${ }^{2,3,4,6}$ is due to the stochastic segregation of mitochondria during embryogenesis; the concentration of damaged mitochondria could differ among patients just due to "chance". In a mitochondrial disease the cellular metabolism is altered (i.e., the ATP is reduced and the concentration of ROS is increased); this could lead to cell death and/or malfunctioning of residual cells.

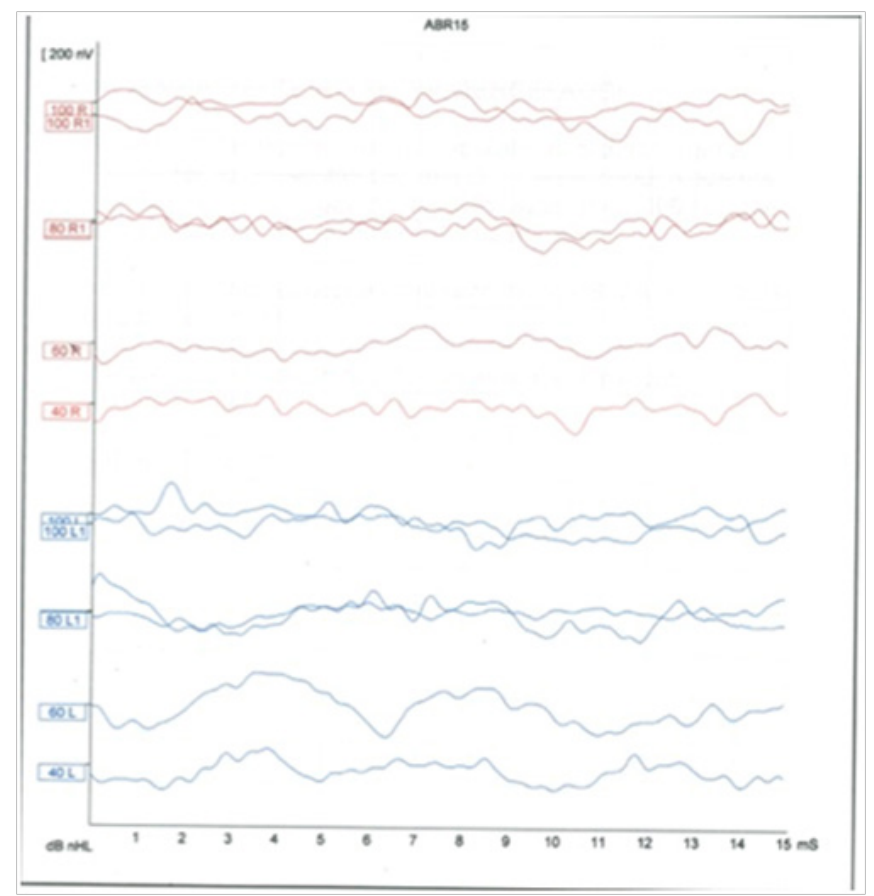

Figure I The image shows the $A B R$ with no structured waves in both sides. The signal is not transmitted at 40 and $60 \mathrm{Db}$, at $100 \mathrm{~dB}$ in the left side seems to appear a wave I. 
Unfortunately, mitochondria are segregated in the cells in a random manner so an electrophysiological test such as ABR may not be sensitive enough to measure the inner ear damage. We are in the process of exploring the viability of using different techniques based on microRNAs specific for hair cells and $\mathrm{SGs}^{12,13}$ for monitoring hearing loss progression. Due to their high sensitivity to detect cells death ${ }^{9}$ and altered function, ${ }^{8}$ microRNAs may provide a valid alternative to traditional ABR test.

\section{Acknowledgments}

None.

\section{Conflicts of interest}

Author declares there are no conflicts of interst.

\section{Funding}

None.

\section{References}

1. Zwirner P1, Wilichowski E. Progressive sensorineural hearing loss in children with mitochondrial encephalomyopathies. Laryngoscope. 2001;111(3):515-521.

2. Kullar PJ, Quail J, Lindsey P, et al. Both mitochondrial DNA and mitonuclear gene mutations can cause hearing loss through cochlear dysfunction. Brain. 2016;139(Pt 6):e33.

3. Santarelli RM, Cama E, Scimeni P, et al. Both mitochondrial DNA and mitonuclear gene mutations can cause hearing loss through cochlear dysfunction. Brain. 2016;140:1-5.el.

4. Sue CM, Lipsett LJ, Crimmins DS, et al. Cochlear origin of hearing loss in MELAS syndrome. Ann Neurol. 1998;43(3):350-359.
5. Chen JN, Ho KY, Juan KH. Sensorineural hearing loss in MELAS syndrome--case report. Kaohsiung J Med Sci. 1998;14(8):519-523.

6. Vandana VP, Bindu PS, Sonam K, et al. Audiological manifestations in mitochondrial encephalomyopathy lactic acidosis and stroke like episodes (MELAS) syndrome. Clin Neurol Neurosurg. 2016;148:17-21.

7. Chisato F, Tatsuya Y. Oxidative Stresses and Mitochondrial Dysfunction in Age-Related Hearing Loss. Oxidative Medicine and Cellular Longevity, . 2014;2014(2014): 6

8. Xue T, Wei L, Zha DJ, et al. miR-29b overexpression induces cochlear hair cell apoptosis through the regulation of SIRT1/PGC-1 $\alpha$ signaling: Implications for age-related hearing loss. Int $\mathrm{J} \mathrm{Mol} \mathrm{Med.}$ 2016;38(5):1387-1394.

9. Someya S, Xu J, Kondo K, et al. Age-related hearing loss in C57BL/6J mice is mediated by Bak-dependent mitochondrial apoptosis. Proc Nat Acad Sci U S A. 2009;106(46):19432-19437.

10. Takahashi K, Merchant SN, Miyazawa T, et al. Temporal bone histopathological and quantitative analysis of mitochondrial DNA in MELAS. Laryngoscope. 2003;113(8):1362-1368.

11. Koda H, Kimura Y, Ishige I, et al. Quantitative cellular level analysis of mitochondrial DNA 3243A > G mutations in individual tissues from the archival temporal bones of a MELAS patient. Acta Otolaryngol. 2010;130(3): m344-m350.

12. Pang J, Xiong H, Yang H, et al. Circulating miR-34a levels correlate with age-related hearing loss in mice and humans. Exp Gerontol. 2016;76:58-67.

13. Di Stadio A, Pegoraro V, Dipietro L, et al. Hearing Impairment in MELAS: new prospective in clinical use of microRNA, a systematic review. Orphanet Journal of Rare Diseases publication in progress. UK. 2010. 